\title{
Los Comentarios Reales del Inca Garcilaso de la Vega: su edición argentina (1943-1944)
}

\author{
Beatriz Colombi ${ }^{1}$
}

Resumen. Este artículo analiza la edición de Ángel Rosenblat de los Comentarios Reales del Inca Garcilaso, publicada por editorial Emecé en Argentina, entre 1944-45. Examina los problemas de la función editor y propone que la edición busca un equilibro entre fidelidad, modernización y mosaico, lo que da por resultado un texto paradigmático para las ediciones del siglo XX que, no obstante, ha sido sometido a revisiones. El primer prólogo, a cargo de Ricardo Rojas, articula un relato fundador de la alianza entre criollos y mestizos, resalta la jerarquía política del texto y consolida su dimensión continental, en diálogo con la crítica garcilasista precedente y futura del texto.

Palabras clave: Comentarios reales; edición; Inca Garcilaso; Ángel Rosenblat; Ricardo Rojas.

\section{[en] The Comentarios Reales of the Inca Garcilaso de la Vega: its Argentine edition (1943-1944)}

Abstract. This article analyzes Ángel Rosenblat's edition of the Inca Garcilaso's Comentarios Reales, published by Emecé (1944-45). It examines the problems of the editor function and postulates a balance between fidelity, modernization and mosaic, which results in a paradigmatic text for editions of the 20th century, not without revisions. In the first prologue, by Ricardo Rojas, the author articulates a founding story of the alliance between creoles and mestizos, highlights the political hierarchy of the text and consolidates its continental dimension, in dialogue with the previous and future criticism of the text.

Keywords: Comentarios reales; Inca Garcilaso; Argentinian edition; Ángel Rosenblat; Ricardo Rojas.

Sumario. 1. El editor: fetichismo, modernización o mosaico; 2. El prologuista y la jerarquía política del text.

Cómo citar: Colombi, B. (2021) Los Comentarios Reales del Inca Garcilaso de la Vega: su edición argentina (1943-1944), en Anales de Literatua Hispanoamericana 50, 159-166.

La edición de la obra del Inca Garcilaso de la Vega ha tenido, sin lugar a dudas, una historia llena de dificultades. Su recorrido azaroso se instala ya desde el título dado por los editores a la segunda parte de los Comentarios Reales en su primera edición de 1617, publicada como Historia general del Perú, aun cuando en su interior figure como Segunda parte de los Comentarios Reales. ${ }^{2}$ En el siglo XVIII, Andrés González de Barcia invierte el orden de aparición de los volúmenes y también su denominación. De este modo, publica primero la segunda parte en 1722 con el título de Historia General, y a continuación, en 1723, la primera parte como Primera Parte de los Comentarios. La censura impuesta a la obra, después de 1780, no impidió su reedición, circulación y nuevas intervenciones editoriales. De este modo, la edición española de 18001801, en trece tomos, basada en la de González de Barcia, sigue un criterio particularmente arbitrario. Con el fin de resaltar el carácter historiográfico de la obra, el anónimo editor resuelve llamar a las dos partes como Historia General del Perú y añadir como subtítulo Comentarios Reales de los Incas, precedido por la conjunción disyuntiva "o", lo cual introduce una alternativa que no estaba presente en el proyecto original. Dice este editor: "[...] aunque se contenta Garcilaso con dar a su luminosa obra el humilde título de

\footnotetext{
${ }^{1}$ Universidad de Buenos Aires. Argentina.

Email: beacolombi@yahoo.com.ar

${ }^{2}$ Luego de los preliminares, comienza el texto con el título LA CONQUISTA DEL PERU. LIBRO PRIMERO DE LA SEGUNDA PARA DE LOS COMENTARIOS REALES de los Incas [...].
} 
Comentarios Reales, a mí me ha parecido, y no sin fundamento, quadrarle más bien el de Historia General del Perú, dexándole también el otro en segundo lugar." (Tomo I: XI). Para reforzar su intención, advierte que el texto no es una novela, aclaración con la que pretende afirmar la veracidad histórica del texto puesta en duda en el siglo XVIII por William Robertson y Cornelius de Pauw. El editor de 1800 suprime la división entre primera y segunda parte, y aún la división en libros, conservando tan solo la partición en capítulos, pues considera innecesarias estas "[...] formalidades arbitrarias e insustanciales, y solo autorizadas por la moda dominante de aquel tiempo" (XII). No satisfecho con estos cambios, reubica en el último tomo, a modo de conclusión, la vida del inca rebelde Inca Inti Cusi Tito Yupanqui, penúltimo inca, tal como había sido contada y documentada por Gabriel de Cárdenas, seudónimo de Andrés González de Barcia, en el prólogo a la segunda impresión, ya "enmendada y añadida" de $1723 .{ }^{3}$ En la misma línea, agrega adiciones al final, "curiosas, y aun casi necesarias", como es el listado de incas, capitanes y virreyes que gobernaron el Perú. En cuanto al aspecto lingüístico, su criterio explícito es seguir las normas de la Real Academia de la Lengua Castellana, tomándose la licencia de "suavizar" algunas voces. Todo resulta extraño en las libertades de este editor que interviene de modo tan flagrante el título, las partes y el orden del texto. Dos siglos más tarde, se puede encontrar en internet una versión digitalizada de la Historia General del Perú fechada en Lima en 2009, realizada por un editor que prefiere mantenerse incógnito y dice esperar como única recompensa "la satisfacción de haber realizado un aporte al ideal sublime del conocimiento libre" (2). ${ }^{4}$ Ambas ediciones (la de 1800, la de 2009) -que elijo como piezas muy dispares aunque significativas de la historia del texto-instalan un enigma pero, al mismo tiempo, iluminan una variable que determina, en buena medida, el sentido de una obra: el editor. Elogioso, aunque invasivo, el de 1800, discreto, aunque innecesariamente secreto, el de 2009.

Este trabajo no pretende ocuparse de la historia editorial de la obra del Inca Garcilaso de la Vega, tarea que ciertamente excede a su extensión y propósito, sino analizar una edición en particular, la publicada en Argentina entre 1943 y 1944. Emprendida por Ángel Rosenblat y publicada por la editorial Emecé de Buenos Aires, ha sido el texto de referencia para posteriores reediciones a lo largo del siglo XX. La primera parte, Comentarios Reales de los Incas, lleva un prólogo de Ricardo Rojas, mientras la segunda, Historia General de Perú (Segunda Parte de los Comentarios Reales de los Incas), reproduce en su introducción el Elogio al Inca de José de la Riva-Agüero, según su versión definitiva de 1936. Este trabajo analiza la función del editor y del prologuista en esta primera edición argentina, que tendrá largo impacto en los estudios garcilasistas.

\section{El editor: fetichismo, modernización o mosaico}

Así como la función autor, señalada por Foucault, la función editor es igualmente determinante para la lectura de un texto. Con su excesiva presencia o declamada ausencia, la intervención del editor es fundamental en el mentado orden de los libros del que nos habla Roger Chartier. Este historiador sostiene que la publicación de los textos implica una pluralidad de agentes y condiciones materiales, de decisiones y mediaciones que definen la forma material de los textos impresos y consecuentemente, su sentido. Toma como ejemplo la puntuación en las ediciones antiguas, desde mediados del XV a comienzos del XIX, y se pregunta a quién se deben atribuir las formas gráficas y ortográficas que estas presentan, para concluir que las mismas correspondían más a los tipógrafos que a los propios autores; también los correctores terciaban en la preparación del manuscrito para la composición y luego en el proceso de corrección de pruebas, al agregar mayúsculas, acentos y puntuación, o normalizar convenciones gráficas, ortográficas o sintácticas (1999, 2002). Estos distintos mediadores (copistas, tipógrafos, correctores, impresores, letrados) preceden y preparan la figura del editor tal como se perfila en el siglo XIX para volverse, en palabras de Chartier, el "[...] coordinador de todas las posibles selecciones que llevan a un texto a convertirse en libro, y al libro en mercancía intelectual, y a esta mercancía intelectual en un objeto difundido, recibido y leído." (Chartier, 1999: 64).

El editor es quien realiza las elecciones que llevan a un texto a convertirse en libro, según las palabras de Chartier, pero también, a un libro a convertirse en otro libro, ya que determinará, a partir de una serie de

\footnotetext{
${ }^{3}$ Gabriel Cárdenas es seudónimo de Andrés González de Barcia Carballido y Zúñiga (Cornejo Polar, 1994: 101).

${ }^{4}$ En línea, http://shemer.mslib.huji.ac.i1/lib/W/ebooks/001531298.pdf
} 
operaciones, la recepción y lectura del mismo. El editor establece la forma del texto, se inmiscuye entre el archivo y su revelación, entre el manuscrito y su paleografía, entre el documento y su desciframiento, entre la edición prínceps y su nueva versión, entre la escritura y la lectura. Su papel es de central trascendencia, sobre todo, cuando pensamos en un archivo disperso, inestable y de extrema fragilidad como el archivo colonial latinoamericano, intervenido por tan distintas voluntades, localizado en tan diversos espacios y publicado en condiciones, generalmente, anómalas.

Ángel Rosenblat (1902-1984) perteneció al selecto grupo de especialistas que se nuclearon en torno a Amado Alonso en el Instituto de Filología de Buenos Aires. Este centro, bajo la dirección de Alonso entre 1927 y 1946, fue considerado una de las instituciones más prestigiosas en los estudios hispánicos a nivel mundial durante esas décadas (Colombi, 2007). En su entorno se reunieron figuras de la talla de Pedro Henríquez Ureña, Eleuterio F. Tiscornia, Ángel Rosenblat, María Rosa Lida, Raimundo Lida, Marcos A. Morínigo, Julio Caillet-Bois, Frida Weber, Ana María Barrenechea, Enrique Anderson Imbert, Luis Jaime Cisneros. Pero este brillante grupo intelectual, así como se potenció en este movimiento centrípeto, también conoció la fuerza del desplazamiento y la dispersión. Atomizada la comunidad que los unía luego de la migración de Amado Alonso hacia Harvard en 1946, muchos de estos investigadores, en la plenitud de su producción, partieron con rumbo a los Estados Unidos, México, Venezuela o Perú, llevando el evangelio de una moderna fe filológica. Ángel Rosenblat, quien había nacido en Polonia, recaló en la Argentina con sus padres inmigrantes, para integrarse, oportunamente, al prestigioso instituto porteño, donde fue discípulo dilecto de Alonso; siguiendo el destino del grupo, parte en 1946 a Venezuela donde trabaja junto a Mariano Picón Salas. Esta red intelectual, que se teje desde el Instituto de Filología, traza un mapa de amistades intelectuales y es responsable del desarrollo de varias vertientes de los estudios literarios y lingüísticos en el continente. Desde la embrionaria estilística que sentaría las bases del estudio teórico de la literatura, hasta la lingüística saussureana, los estudios hispánicos e hispanoamericanos, la historia cultural y la edición crítica. En este semillero de riguroso trabajo intelectual, la edición de Ángel Rosenblat estuvo orientada al rescate del archivo colonial. ${ }^{5}$

Pertenecer al Instituto de Filología era un blasón para un intelectual de su tiempo. Por eso Rosenblat lo exhibe en la portada de la publicación del libro donde consta: "Edición al cuidado de Ángel Rosenblat del Instituto de Filología de la Universidad de Buenos Aires". Ser un reconocido filólogo y lingüista era una garantía para la tarea, pero además otras circunstancias confluyeron. Por una parte, el interés en el mundo colonial de Ricardo Rojas, historiador de la literatura argentina, decano de la Facultad de Filosofía y Letras entre 1922-1926 y rector de la Universidad de Buenos Aires entre 1926-1930, decidido constructor de mitos nacionales y adalid de la educación como vía de unificación social, en la línea de los ensayistas latinoamericanos que González Echevarría ha llamado "la voz de los maestros". Por otra parte, la expansión de la industria editorial en Buenos Aires, consecuencia de la migración española durante la guerra civil, con el desarrollo de dos empresas líderes como Losada y Emecé, permitió llevar a cabo estas aventuras editoriales donde los clásicos y los nuevos encontraban posibilidades de circulación nacional y continental. Ignoramos donde surgió el proyecto de la publicación de los Comentarios: si en Rosenblat, Alonso o Rojas. En la búsqueda de documentos y correspondencia, solo encontré algunas breves esquelas entre Rosenblat y Rojas, que aluden a las pruebas de galera de los tomos.

Antes de emprender la edición de los Comentarios, Rosenblat ya había cuidado de la edición del Amadís de Gaula (Losada, 1940) e Historia de los incas de Pedro Sarmiento de Gamboa (1942), y se encargará a continuación de las Cartas completas de Lope de Vega (1948) y Viajes al Estrecho de Magallanes de Pedro Sarmiento de Gamboa (1950), todos para editorial Emecé. Con esta nutrida producción y dedicación al tema, es lícito que nos preguntemos cuáles eran sus principios en tanto editor. En 'Fetichismo editorial', incluido en el artículo "Fetichismo de la letra" de 1948, Rosenblat reflexiona sobre los problemas que presenta la edición de documentos y libros antiguos. ${ }^{6}$ Sostiene que la tarea del editor alterna entre mantener el texto en su integridad o proceder a su modernización. Esta alternativa entre fidelidad y libertad recuerda a las tensiones del traductor que plantea Walter Benjamin en "La tarea del traductor". De hecho, editor y traductor realizan una tarea semejante de traspaso y transcodificación, que implica un compromiso ético con una palabra "original". En el artículo que aludimos, Rosenblat habla del "editor fetichista", fiel a ultranza, que reproduce

\footnotetext{
5 Amado Alonso promovió desde el Instituto de Filología la Biblioteca de Dialectología Hispanoamericana (1930), la Colección de estudios indigenistas (1931) y la Colección de Estudios Estilísticos (1932) además de la Revista de Filología Hispánica, luego continuada en México como Nueva Revista de Filología Hispánica.

${ }^{6}$ Ángel Rosenblat, "Fetichismo de la letra”, Revista Nacional de Cultura, nº. 66, 1948, 31-52; reunido luego en Ángel Rosenblat (1977).
} 
"hasta las erratas de imprenta del texto original" (1977: 87), mientras que el editor modernizador, es aquel que adapta todo a los criterios contemporáneos. Entre la fidelidad absoluta y la actualización hasta las últimas consecuencias, Rosenblat alude a una tercera posibilidad, que llama el "mosaico ortográfico", resultante de la fusión de criterios editoriales de distintas épocas los que, sumados, dan por resultado una miscelánea de intervenciones datadas sobre el texto. Coloca como ejemplo de este último caso, una obra del siglo XIII en la que pesan la superposición de tres temporalidades: "La edición resulta así un mosaico ortográfico: obra del siglo XIII, publicada en 1944 con la ortografía de 1850" (88). Estos tres criterios sobrevuelan la edición del impreso antiguo: fetichismo, modernización o mosaico. Proponemos que Ángel Rosenblat hace una combinación y equilibrio entre estas tres opciones en los Comentarios Reales.

En el "Criterio de esta edición", incluido en el final de la primera parte de los Comentarios Reales, Rosenblat informa que sigue la edición prínceps de Lisboa, 1609, y resume cuáles han sido sus decisiones editoriales (Garcilaso de la Vega, 1943 II: 299-306). Opta por una modernización que normaliza las abundantes oscilaciones ortográficas propias de la época, pero advierte: "Conservamos rigurosamente, en cambio, todas las diferencias ortográficas y particularidades que representan la pronunciación del siglo XVI" (299). Por ejemplo: respeta la incidencia de $s$ y $s s, x$ y $j, z$ y $c, b$ y $v$, en la medida que representan sonidos diferenciados en el 1600. En cuanto a la acentuación -casi inexistente o errática en el original- elige actualizarla por completo. Así como la puntuación, que se ajusta a las normas contemporáneas de la estructura de la frase: "Nos hemos atenido a las normas actuales que responden mejor a la estructura gramatical de la frase" (299). Introduce también marcas tipográficas inexistentes en el texto de 1609, como comillas, apóstrofe, cursivas y corchetes correctivos de erratas o desarrollos. Del mismo modo, regulariza el uso de mayúscula y minúscula, excepto para sustantivos que tienen "jerarquía especial" en lo que llama el "sistema del autor", como Rey, Inca, Sol, Príncipe, Emperador, así como en los nombres indígenas. Si bien, como ha señalado José Antonio Mazzotti (1996), este proceder no es siempre constante, así Rosenblat coloca con mayúsculas "Nuestro Padre el Sol" (Cap. XV, Libro I), mientras, en la edición príncipe figura en minúscula "nuestro padre el Sol", en evidente contagio con la plegaria "Padre Nuestro", direccionando de este modo el sentido del texto.

Dos principios generales guían la mayoría de las opciones de Rosenblat. Lo que llama el "sistema del autor" y la prosodia, tanto la general del siglo XVI, como la particular del Inca, cuya pronunciación intenta imaginar, en una aproximación casi ficcional a su locución. Así, por ejemplo, cuando se refiere a la letra "h" que tiene un uso altamente irregular en el texto, y en la época, resuelve mantenerla en algunas oportunidades a pesar de su incorrección ortográfica ya que, según su parecer, el Inca "quizás la aspiraba" por su carácter de quechua hablante (300). O resuelve modernizar la grafía latinizante de "Christo", pero no así de "Chancillería", porque "es posible que la ch se pronunciase" (300). Contempla también particularidades del quechua, como cuando dice: "El quechua carecía del sonido $b, v$. Por esta razón transcribimos con $u$ todos los casos en que aparece la grafía $v$ " (300). Su criterio, explicado minuciosamente, pretende la construcción de lo que podemos llamar, tomando un término propio del mundo jurídico, un texto consolidado, ${ }^{7}$ donde el lector tuviese la posibilidad de "delimitar lo antiguo y lo moderno" (299). Salva también las erratas de la edición de 1609 que son advertidas, la mayoría de las veces, en la Fe de erratas del original: "Algunas se deben a la tipografía portuguesa (primeiro, batalha), otras a la dificultad de transcribir voces y nombres indígenas" (302).

Proponemos que Rosenblant aspiraba a realizar una edición trans-histórica, cuya validez pudiera vencer tanto el anacronismo de la fijación como el desgaste del paso del tiempo. Incurre, de este modo, en una de las fantasías del editor: ser el oficiante de una ceremonia de transparencia y pase, guiado por la máxima fidelidad a los principios a los que adscribe. Pero, como sabemos, se trata de una utopía lingüística, ya que todo cambio, por mínimo que sea, introduce significaciones nuevas. Antonio Mazzotti ha señalado las modificaciones en la puntuación que introdujo Rosenblat, quien adapta la pausa retórica y oral de la edición prínceps a un patrón prosódico que responde a un carácter gramatical moderno. Este proceder altera la visibilidad -según la conocida hipótesis de este crítico-, del subtexto andino de la obra (1996). Paradojalmente, ambas perspectivas, tanto la de Rosenblat como la de Mazzotti, están relacionados con la performance oral. Rosenblat se detiene en la acentuación y entonación fonemática, Mazzotti en el ritmo o patrones rítmicos que construyen las pausas en el discurso.

${ }^{7}$ Un "texto consolidado o refundido", según el Diccionario de la Real Academia Española, en el ámbito del Derecho significa: "texto que integra en un único cuerpo legal sistemático varias leyes anteriores y sus sucesivas modificaciones". 
Hacia los mismos años en que Rosenblat emprende esta edición en Buenos Aires, Jorge Luis Borges escribía en esta ciudad "Pierre Menard, autor del Quijote", bajo el signo del mismo campo intelectual. Borges propone en este cuento que todo texto se transforma con las sucesivas generaciones de lectores en la medida en que se modifican los dispositivos de lectura, independiente de las variaciones efectivamente introducidas en la materialidad de la escritura. Para decirlo con sus palabras: "el texto cambia como el río de Heráclito." Este pareciera ser un callejón sin salida o la paradoja elemental de toda edición: el tiempo pasa para el texto, para la lengua y para la comunidad de lectores, como mecanismos encadenados y al mismo tiempo independientes entre sí. La edición porteña de 1943 y 1944 nace de una acumulación de saberes que Rosenblat ha atesorado hacia esa época, de carácter lingüístico, filológico, histórico y culturalista, que se volcará también en varios de sus ensayos.

\section{El prologuista y la jerarquía política del texto}

Entre el archivo, el manuscrito, la edición prínceps, y la edición contemporánea, dijimos, media el editor. Pero en otro nivel, encontramos otra intervención igualmente significativa, el prologuista, orientador de la lectura y puente entre el texto y su lector. El prólogo, como enseña Genette, es un paratexto y como tal: "Más que de un límite o de una frontera cerrada, se trata aquí de un umbral o -según Borges a propósito de un prefacio-, de "un 'vestíbulo' que ofrece a quien sea la posibilidad de entrar o retroceder." (2001: 7). En su breve y programático prólogo a la primera parte de los Comentarios Reales, Ricardo Rojas (1882-1957) exhibe sus antecedentes para escribir sobre el tema. Se ha ocupado del Inca Garcilaso en sus clases universitarias, en su Historia de la literatura argentina, en el congreso de Americanistas realizado en Lima en 1939. Desde las primeras décadas del siglo XX, y luego de la decisiva tesis de Riva-Agüero, el Inca había sido investido de los más diversos atributos y propiedades. Síntesis del "peruanismo" para Riva-Agüero, "primer criollo" para Luis Albero Sánchez, "profeta" de indios, mestizos y criollos y primer escritor de América para Luis E. Valcárcel, entre otros juicios que enaltecieron la figura y lugar de Garcilaso. Ricardo Rojas manifiesta su declarada "devoción" por el tema, que lo ha llevado a visitar su casa natal en el Cuzco y a conocer su tumba en la Catedral de Córdoba, cuyo epitafio, redactado por el autor andino, reproduce en el comienzo del trabajo. En su viaje a Europa de 1908, viaje iniciático o grand tour que realizaban todos los escritores de su tiempo, relatado en Retablo español (1938), Rojas cuenta cómo intentó adquirir una primera edición de los Comentarios en un anticuario de Sevilla, pero el tomo terminó escurriéndose de sus manos debido el escaso capital de viajero. Escribir este prólogo de 1943 es una segunda oportunidad de hacerse simbólicamente del libro. El rescate del pasado será una preocupación central para Rojas, comprometido por encontrar un lugar de legitimación para los estudios coloniales, particularmente en la Argentina, donde no tenían el brillo ni los antecedentes de los que ya gozaban en Perú o en México. Una de sus primeras publicaciones en este campo fue exhumar los manuscritos del llamado primer poeta argentino, Luis de Tejeda (Colombi, 2014). En el volumen de la Historia de la literatura argentina, destinado a Los coloniales, Rojas expone la necesidad de reconstruir ese pasado para encontrar "lo larval" de las sociedades americanas.

En el prólogo a Comentarios, Rojas realiza un decidido gesto de consolidación de este archivo literario colonial. Una decidida estrategia, en este sentido, es relacionar la obra del Inca Garcilaso con la independencia americana y con una figura señera como José de San Martín, quien había propuesto la publicación de esta obra. ${ }^{8}$ La unión del héroe militar (San Martín) y del héroe cultural (Inca Garcilaso), le permite articular un relato de origen que será importante para la circulación del texto en un espacio nacional y, al mismo tiempo, a nivel continental. Rojas promovía la unión de raíces hispánicas con el pasado aborigen en postulaciones que resultaron impactantes en su momento, si bien que se tornaron débiles e inviables con el paso del tiempo; por ejemplo, el concepto de "eurindia", sostenido en el ensayo homónimo de 1924. En la Casa Museo Ricardo Rojas de Buenos Aires, la sala que precede al estudio del escritor, lleva precisamente el nombre de "sala incaica" y está decorada con diseños alusivos. La casa, de 1927, fue encargada por Rojas al arquitecto Ángel Guido, quien comulgaba con la ideología de Eurindia y se propuso plasmarla en una arquitectura neocolonial donde el mensaje nacionalista se entreveraba con el rescate del pasado prehispánico. La casa de Rojas reúne, como un aleph, los distintos momentos de América (prehispánica, colonial, independentista, nacional), fusión a la que aspiraba su programa. ${ }^{9}$ La edición de Comentarios reales e

\footnotetext{
${ }^{8}$ Rojas publicó en 1933 su conocida biografía del liberador, El santo de la espada.

${ }^{9}$ Véase Bonicato 2010-2011.
} 
Historia General de Perú de 1943 y 1944, respectivamente, obedece en su tipografía y presentación a esta estética neocolonial con la reproducción de las xilografías de la edición de Madrid de 1723.

Hispanismo, nacionalismo y criollismo (muchas veces renegados, como en el caso de Borges) conformaron el tramado ideológico de la generación de Rojas, algunos de cuyos integrantes buceaban en el archivo colonial en busca de respuestas a los enigmas de la cada vez más evanescente y peregrina identidad. Podría pensarse que Rojas enarbola la misma estrategia hispanista y aristocratizante esgrimida por RivaAgüero en Perú, pero si lo hace es con sustantivas diferencias en cuanto a sus respectivas concepciones del nacionalismo y aun del indigenismo. ${ }^{10}$ Respecto al indigenismo, Rojas profesó un interés persistente en las raíces incásicas a las que dedicó tanto estudios académicos como obra creativa. Rojas perteneció a la generación del 900, estuvo próximo al modernismo, se afilió a un pensamiento nacionalista, democrático, laico e inclusivo - a diferencia de otras vertientes nacionalistas conservadoras y xenófobas-, militó en el Partido Radical y sufrió cárcel y persecución después del golpe de 1930; se plegó a la Revolución Libertadora de 1955, como buena parte de la elite intelectual de su tiempo. ${ }^{11}$

Rojas construye en el prólogo una ficción de origen, donde nos muestra a San Martín, debilitado por la enfermedad, que hace reposo en las sierras de Saldán, Córdoba, hacia 1814. El Libertador sostiene allí tertulias con sus continuos visitantes y concibe la idea de una reedición de los Comentarios Reales, para acompañar su campaña de independencia política. La obra, que se financiaría con suscripciones, sería publicada en Londres, lo que finalmente no sucede. Pero, aunque la intención queda trunca, Rojas recoge esta anécdota (documentada y debidamente probada) para fundar en ella una triple articulación. Una, la fusión entre la política y las letras; dos, la superación de los límites nacionales y la proyección de una mirada continental, y tres, la unión de un representante del sector criollo, como San Martín, con un mestizo, como el Inca. Me interesa remarcar la primera operación. Más allá de sus conocidos méritos historiográficos, literarios o filológicos, a Rojas le interesa destacar el carácter político de la obra del Garcilaso, a quien consideraba promotor de la unión de los heterogéneos sectores coloniales y cimiente de la rebeldía que alentó Tupac Amaru II en 1780, lo que motivó la prohibición de la obra por Real Cédula de Carlos III del 21 de abril de 1782, ya que despertaba "la conciencia histórica de los pueblos autóctonos" (Tomo I: XI). Rojas sostiene que San Martín "ratifica esa condición del libro, al haberse adoptado una teoría de la reivindicación nativista que acercaba a los criollos y a los indios para formar un solo frente contra el sistema virreinal" (X). Rojas considera que, en virtud de estos dos episodios en torno a los Comentarios, el protagonizado por Tupac Amaru en el XVIII y por San Martín en los albores del XIX, "el libro del inca Garcilaso tiene en la historia americana una elevada jerarquía política, superior, acaso, a la que le han reconocido eminentes críticos por su valor arqueológico y literario" (X, la cursiva es mía). De este modo, Rojas alimenta una línea de lectura que será profundizada por José Durand, Alberto Flores Galindo, Antonio Cornejo Polar, Carlos García Bedoya, David Brading, Julio Ortega, José Antonio Mazzotti, entre otros críticos. ${ }^{12}$

Ricardo Rojas engarza dos tradiciones de lecturas críticas sobre los Comentarios. En una de ellas, encontramos al Inca Garcilaso como signo de fusión y síntesis armónica de culturas, lo que apunta a la construcción de un discurso de homogeneidad nacional, como lo ha señalado Cornejo Polar a partir de su examen de la intencionada rehabilitación de José de la Riva-Agüero. La otra, en cambio, enfatiza en el poder resistente y cuestionador de los Comentarios hacia la conquista y el orden colonial. Según esta última, bajo una retórica humanista de aparente concordia, subyace un mundo de "conflictos y alteridades" para Cornejo Polar, un "discurso disidente" para Carlos García Bedoya, o las resonancias de un "subtexto andino" para José Antonio Mazzotti. Cornejo Polar señaló el "riesgo de las metáforas" (1998) de las lecturas transculturadoras que ofrezcan una síntesis apacible de los conflictos. Propuesta que podemos relacionar con lo sostenido por Hommi Bhabha en relación a los tropos en la literatura colonial. Para Bhabha, la percepción de las figuras del discurso colonial como metáforas impone una lectura universalista, porque la metáfora no hace concesiones a la especificidad cultural de los textos. En cambio, la metonimia, vuelve al texto sintomático de las fuerzas y contradicciones sociales, culturales y políticas que lo atraviesan (1984). En la obra del Inca reconocemos ambos movimientos: uno metafórico de concordia y totalidad y otro, metonímico, de diferenciación y fragmento (Colombi, 2016). Esa hendidura ya aparece en las primeras aproximaciones

\footnotetext{
${ }^{10}$ Se conocieron y mantuvieron amistad y correspondencia. En carta de 1944, Riva-Agüero agradece las citas de Rojas en su prólogo al libro, en los ejemplares que acaba de adquirir de la obra, supuestamente, en Lima.

${ }^{11}$ Véase presentación de Darío Pulfer a Ricardo Rojas (2010).

${ }^{12}$ Julio Ortega sostiene que es el discurso político el que organiza a los Comentarios Reales en "El Inca Garcilaso y la política del discurso" (1992).
} 
críticas en el siglo XX, y si bien es embrionaria en el texto de Rojas, es significativo que establezca como eje de su lectura la elevada jerarquía política del texto dando, además, un giro al universo de pertenencias genéricas atribuidas a la obra: comentario, historia, novela utópica, tragedia, y ahora, texto político.

Rojas defiende al Inca de la lectura intencionada de Marcelino Menéndez Pelayo, quien considera a los Comentarios una novela utópica y "leyenda incásica", fruto según las palabras del crítico español, de un "alma crédula, supersticiosa" con deficiente "discernimiento crítico". Esta lectura ya había sido rebatida por José de la Riva-Agüero quien, recordemos, desplaza la tonalidad de la obra hacia la tragedia, así habla de los Comentarios como "clásica" y "desgarradora" tragedia, sugiriendo un tipo de tramado de la historia que nos aproxima a la conocida propuesta de Hayden White respecto a los tropos que sostienen la narrativa histórica (2003). Rojas llama a concluir con el "prejuicio de considerar a Garcilaso un novelista porque es buen escritor, o de considerarlo historiador parcial cuando trata de los Incas porque él es Inca, y cuando trata de las guerras civiles de los españoles en el Perú, porque su padre perteneció a uno de los bandos en lucha." (XVII). Toca así en nudos de la crítica garcilasista que aparecen y reaparecen en muy diferentes momentos de su recepción: la construcción narrativa, las oscilaciones del relator, la defensa inamovible de la figura paterna y el orgullo de su genealogía materna. Coloca también en perspectiva la obra del Inca Garcilaso al decir que brilló solitaria durante mucho tiempo, mientras que hacia 1940 ya puede ser considerada junto con otras piezas rescatadas del archivo. Si bien resalta que "Garcilaso aventaja a sus congéneres, en cultura, en alientos, en ingenio" y en "talento literario", comenta también los "nuevos documentos de escritores mestizos", como Guamán Poma de Ayala, valorando de este modo un campo de estudio que en ese momento estaba en desarrollo y que alcanzará importantes dimensiones décadas más tarde (XIX). Marca, en este sentido, una diferencia crítica respecto a Riva-Agüero quien desdeña, displicente, estos materiales nacidos de "modestos auxiliares", "sin inteligencia, criterio ni sintaxis", según dice en su Elogio al Inca.

Rojas agrega la inflexión autobiográfica a su prólogo, así confiesa que el Inca ha motivado sus propias elecciones intelectuales: "Con su lectura empezó mi devoción personal por Garcilaso, a quien mucho debo en mis estudios indófilos." (XIX). Por último, Rojas eleva a Garcilaso a verdadero fundador de la "conciencia americana", como la figura augural de un trayecto que desemboca en la independencia sanmartiniana. La operación crítica de Rojas es construir un clásico, un "clásico de América" como dirá José Durand, pocos años más tarde.

Pedro Henríquez Ureña incluye al Inca Garcilaso en su canon de infaltables de la literatura hispanoamericana en "La América Española y su originalidad" ${ }^{13}$, de 1936, y lo incorpora como objeto de estudio en los programas de literatura hispanoamericana que dicta en la Universidad Buenos Aires en esos años. En la estela de estos aportes, hacia 1949 el investigador argentino Luis Arocena publica en Buenos Aires El Inca Garcilaso y el humanismo renacentista, aporte a la construcción de esta importante veta en la tradición crítica garcilasista, en la que podemos encolumnar a José Durán, Julio Ortega, Margarita Zamora, Carmen Bernand, José Antonio Mazzotti, entre otros especialistas. Rehabilitado, reivindicado y peruanizado por Riva-Agüero quien, como dice Cornejo Polar, convierte al Inca en "gran signo y emblema de la nación" (1994: 104), su obra adquiere en los discursos de las décadas del 30 y 40 una dimensión continental, de la que abreva y a la que a su vez alimenta el texto de Ricardo Rojas. Por eso Rojas atribuye a esta edición la misión ser una "fuente de unidad" americana, es decir, le otorga un cometido político cultural. Si Rojas imprimió este carácter a su prólogo, Rosenblat impulsó una versión moderna y legible para su época, que se constituyó en la edición americana distintiva del siglo XX. Sin llegar al postulado de Pedro Henríquez Ureña que sostenía que cada generación debe traducir a su Homero, el Inca merecerá seguramente otra edición en el siglo XXI. O quizá, guiados por la ilusión de un tiempo vacío e imposible, con un gesto a lo Pierre Menard, volvamos a leer las versiones prínceps de 1609 y 1617 , en formato digital.

\section{Referencias bibliográficas}

Bhabha, Homi (1984), "Representation and the Colonial Text: A Critical Exploration of Some Forms of Mimeticism", en Frank Gloversmith (ed.). The Theory of Reading (págs. 93-121). Sussex: Harvester.

Bernand, Carmen (2006). Un Inca platonicien: Garcilaso de la Vega. Paris: Fayard.

${ }^{13}$ Leída en la Séptima Conversación de la Organización de Cooperación Intelectual que se realizó en Buenos Aires entre el 11 y el 16 de septiembre en 1936. 
Bonicato, Virginia. (2010-2011), "La materialización de una imagen nacional: Ricardo Rojas en la arquitectura argentina", Boletín de Estética, año VI, nº 15, diciembre-marzo, págs. 3-29.

Brading, David A. (1991), "Un humanista inca", en Orbe Indiano. De la monarquía católica a la república criolla, 1429-1867. México: Fondo de Cultura Económica, págs. 283-301.

Colombi, Beatriz (2007), “Amado Alonso en Buenos Aires. Inserciones en un campo intelectual”, en Juana Martínez (ed.). Exilios y residencias. Escrituras de España y América. Madrid: Iberoamericana, págs. 55-66.

- (2014). "Luis de Tejeda y la Babilonia de las Indias", en Noé Jitrik, Cristina Iglesia y Loreley El Jaber (dirs.). Historia Crítica de la Literatura Argentina. Volumen I: Una patria literaria. Buenos Aires: Emecé, págs. 265-291.

(2016), "Del reinar al vasallaje: armonía y despojos en los Comentarios Reales", Cuadernos Americanos. Nueva Época, vol. 3, no. 157, México, págs. 51-62.

Cornejo Polar, Antonio (1994). Escribir en el aire. Ensayos sobre la heterogeneidad socio-cultural en las literaturas andinas. Lima: Horizonte.

Literaria Latinoamericana, 47, págs. 7-11.

Chartier, Roger (1994). The order of books. Readers, Authors, and Libraries in Europe between the Fourteenth and Eighteenth Centuries. Standford: Standford University Press.

--------, ------ (1999). Cultura escrita, literatura e historia. Conversaciones con Roger Chartier. México: Fondo de Cultura Económica.

------, ---- (2002), “A mediação editorial”, en Os desafíos da escrita. São Paulo: UNESP.

García-Bedoya, Carlos (2000). La literatura peruana en el período de estabilización colonial. Lima: Universidad Mayor de San Marcos.

Durand, José (1976). El Inca Garcilaso, clásico de América. México: Sep-Setenta.

Flores Galindo, Alberto (1986). Buscando un Inca. Identidad y utopía en Los Andes. La Habana: Casa de las Américas.

Garcilaso de la Vega, Inca (1800-1801). Historia General del Perú ó Comentarios Reales de los Incas. Tomo I-XIII. Madrid: Imprenta de Villapando.

---on (1943-1945). Comentarios Reales de los Incas. Tomo I-II. Edc. de Ángel Rosenblat. Pról. de Ricardo Rojas. Buenos Aires: Emecé Editores.

(1944). Historia General de Perú (Segunda Parte de los Comentarios Reales de los Incas).

Tomo I-III. Ed. de Ángel Rosenblat. Elogio del autor y examen de la segunda parte de los Comentarios Reales por José de la Riva-Agüero. Buenos Aires: Emecé Editores.

Genette, Gérard (2001). Umbrales. México: Siglo XXI.

Mazzotti, José Antonio (1996). Coros mestizos del Inca Garcilaso. Resonancias andinas. Lima: Fondo de Cultura Económica.

Ortega, Julio (1992), "El Inca Garcilaso y la política del discurso”, en El discurso de la abundancia. Caracas: Monte Ávila, págs. 29-36.

Rodríguez Garrido, José A. (1995), "La identidad del enunciador en los Comentarios Reales”, Revista Iberoamericana, LXI, 172/173, págs. 371-383.

Rojas, Ricardo (2010). La restauración nacionalista. Presentación de Darío Pulfer. La Plata: UNIPE-Editorial Universitaria.

Rosenblat, Ángel (1977). Sentido mágico de la palabra. Caracas: Universidad Central de Venezuela.

--------, --- (2002). El español en América. Sel., pról., cronología y bibliografía de María Josefina Tejeda. Caracas: Biblioteca Ayacucho.

Serna, Mercedes (2017); "El proyecto político del Inca Garcilaso de la Vega y la legislación colonial española", Revista de crítica literaria latinoamericana, XLIII, 85, págs. 275-286.

White, Hayden (2003), "Tropología, discurso y modos de conciencia humana", en El texto histórico como artefacto literario. Barcelona: Paidós, págs. 63-105.

Zamora, Margarita (1987), "Filología Humanista e historia indígena en los Comentarios Reales", Revista Iberoamericana, 53, 140, págs. 547-558. 